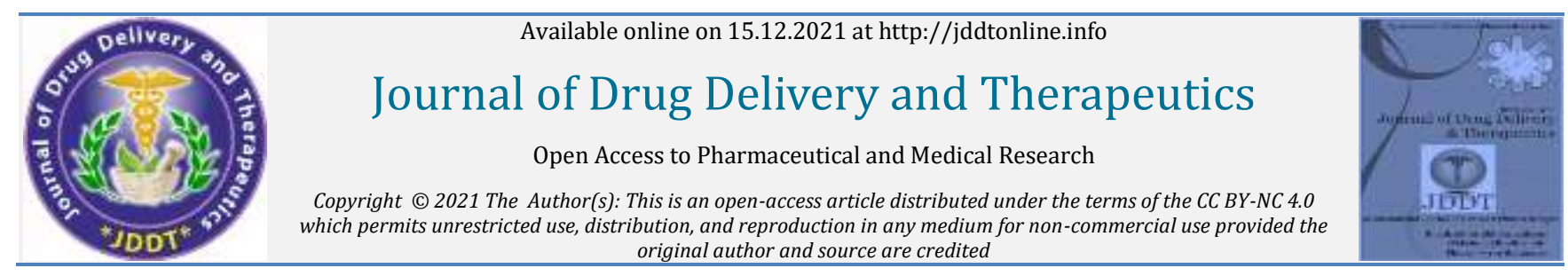

Open Access Full Text Article original author and source are credited

Review Article

\title{
In-Situ Ocular Gel Pharmaceutical Delivery System: A Recent Review
}

\author{
Kapila Sohan*1, Dev Dhruv ${ }^{1}$, Prasad D.N. ${ }^{2}$ \\ ${ }^{1}$ Department of Pharmaceutics, Shivalik College of Pharmacy, Nangal, Punjab, India \\ ${ }^{2}$ Department of Pharmaceutical Chemistry, Shivalik College of Pharmacy, Nangal, Punjab, India
}

\section{Article Info:}

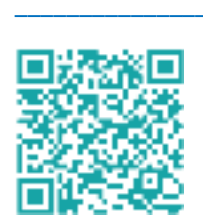

\section{Article History:}

Received 08 October 2021

Reviewed 16 November 2021

Accepted 25 November 2021

Published 15 December 2021

\section{Cite this article as:}

Kapila S, Dev D, Prasad DN, In-Situ Ocular Gel Pharmaceutical Delivery System: A Recent Review, Journal of Drug Delivery and Therapeutics. 2021; 11(6-S):173-180

DOI: http://dx.doi.org/10.22270/jddt.v11i6-S.5098

*Address for Correspondence:

Kapila Sohan, Department of Pharmaceutics, Shivalik

College of Pharmacy, Nangal, Punjab, India

\author{
Abstract
}

\begin{abstract}
Ocular Drug Delivery has been a key challenge and attractive field for the pharmaceutical scientist due to peerless anatomy and physiology of eye. Glaucoma, dry eye syndrome, keratitis, endophthalmitis, trachoma, and conjunctivitis are just a few of the conditions that can affect the eye. In order to accomplish efficient ocular treatment within the eye, At the point of action, an appropriate supply of active substances must be given and sustained. Due to fast precorneal medication loss, traditional treatment has a low bioavailability. The bioavailability of a medicine is also influenced by static and dynamic barriers. To address the limitations of traditional treatment, significant efforts are being made to develop innovative medication systems for ocular delivery. When a drop is injected into the eye, it goes through a sol-gel transition and forms a cul-de-sac. The in-situ gel system, which comprises thermally triggered, $\mathrm{pH}$ triggered, and ion cross linking systems, is the subject of this review. It includes a step-by-step procedure for preparing the $\mathrm{pH}$-triggered system as well as assessment parameters.
\end{abstract}

Keywords: Conventional dosage form, Anatomy and physiology in eye, In-situ gel.

\section{INTRODUCTION}

The ocular medication delivery system is important and complex since the human eye is an isolated organ where drug administration is difficult. Furthermore, due to quick and widespread removal of medicines from pre-corneal lachrymal fluid via solution drainage, lachrymation, and nonproductive absorption by conjunctiva, traditional ophthalmic formulations have a short pre-corneal residence period and low bioavailability ${ }^{1}$. Various attempts have been made to manufacture stable sustained release in situ gels in order to overcome the difficulties associated with standard ophthalmic formulations. Newer research in ophthalmic drug delivery systems is focusing on incorporating a variety of drug delivery technologies, such as developing systems that not only lengthen the vehicle's contact duration with the ocular surface, but also slow the drug's removal. The in-situ gel system is manufactured as a liquid preparation for instillation into the eyes that converts to gel when exposed to the physiologic environment, extending the delivery system's precorneal residence duration and improving the drug's ocular bioavailability ${ }^{2}$. The production of gels is influenced by variables such as a change in a certain physio-chemical parameter $(\mathrm{pH}$, temperature, ion-sensitive) that allows the drug to be delivered in a controlled and sustained way. In situ gel, nanosuspension, nanoparticulate system, liposomes, dendrimers, ocular iontophoresis, collagen shield, minidisc, ocular film, implants, and other innovative dosage forms are among them ${ }^{3}$. Because of the limitations of the ocular route, such as non-productive absorption, drug impermeability to the cornea, drainage, induced lachrymation, and tear turns over, developing ocular drug delivery devices has always been difficult. Topical medicine application to the eye is a well-established method of administration for the treatment of a variety of ocular illnesses such as dryness, conjunctivitis, keratitis, and eye fever. Polymers, which play a significant role in drug delivery to the pre and intra ocular tissues, have been studied as new techniques for drug administration to the eye ${ }^{4}$. Such consistent efforts have resulted in an improvement in bioavailability and an extension of the therapeutic effect of an eye medication. Smart polymeric systems have shown to be a potential method of medication delivery. After being delivered, these polymers go through a sol-gel transition. Before administration, they are in the solution phase, but under physiological conditions, they gel. The pharmaceuticals' ocular bioavailability can be increased by enhancing their corneal permeability and lengthening their residence duration in the cul-de-sac 5. This article provides a brief overview of in situ gels, their assessment, and conventional dose forms, as well as anatomy and physiology in the eye. 


\section{CONVENTIONAL DOSAGE FORM}

Topical administration is frequently chosen over systemic administration for eye illnesses in order to minimize systemic toxicity, have a faster start of effect, and reduce the necessary dosage. Though topical administration has numerous benefits for treating illnesses of the anterior structures of the eye, it has the major drawback of limited bioavailability due to a number of biological mechanisms (Fig. 1) that exist to protect the eye and hence limit the entry of ocular medications. The limitations of topical ocular administration are mentioned below ${ }^{6}$.

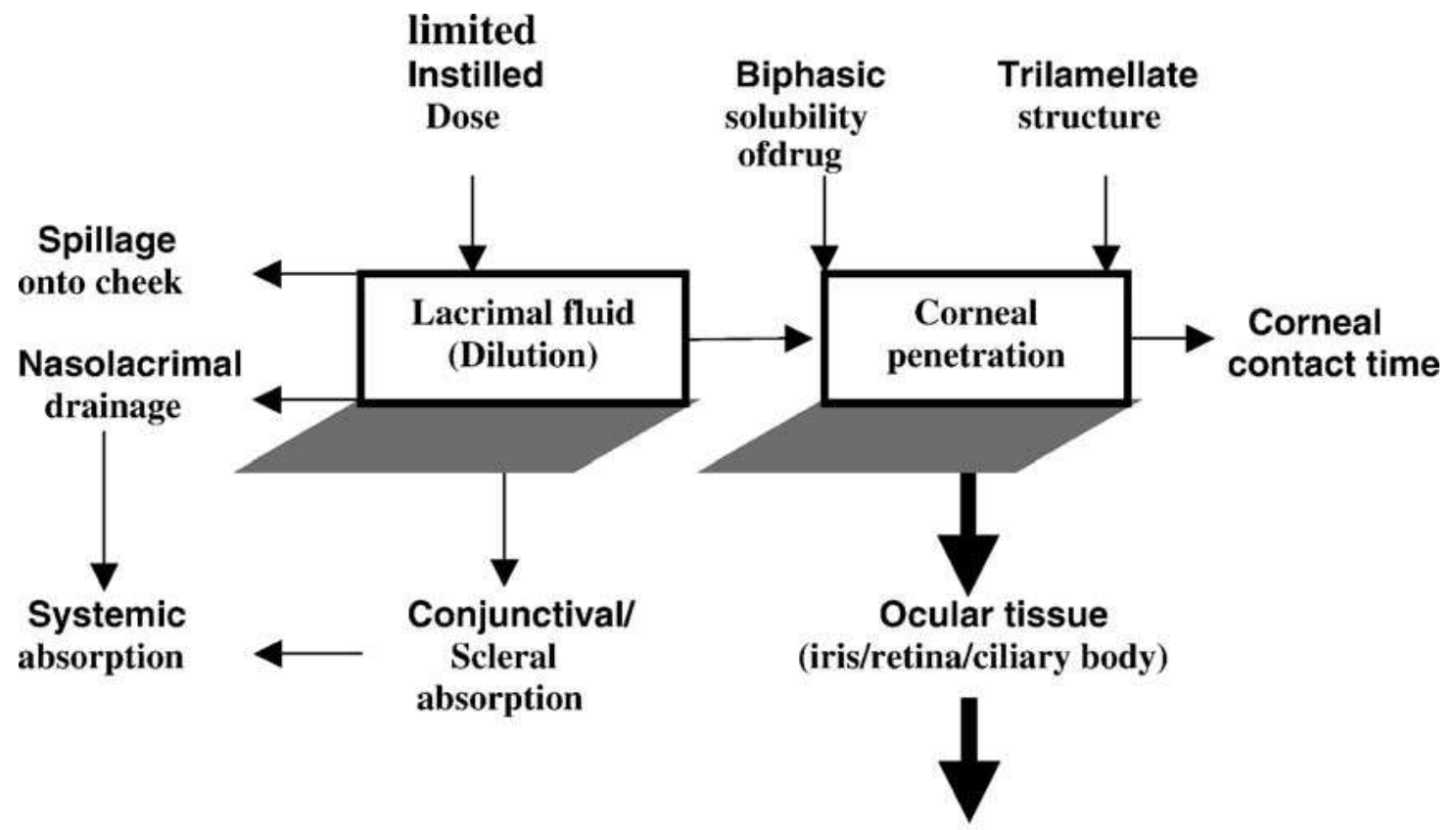

Physiological response

Figure 1: Factors attributing to poor bioavailability of an ophthalmic formulation 6

\section{CORNEAL ABSORPTION}

The cornea is the primary pathway for intraocular absorption (Ahmed and Patton, 1987). The cornea's modest surface area and relative impermeability are two characteristics that make it an excellent barrier to drug absorption. In humans, however, the conjunctiva, a vascular thin mucous membrane that covers the inside of the eyelids and the anterior sclera, is around 17 -fold bigger than the cornea ${ }^{7}$. Furthermore, it is between 2 to 30 times more drug permeable than cornea. As a result, conjunctival drug absorption is a significant loss factor that competes with corneal absorption following topical application to the preocular region ${ }^{8}$. Second, in terms of drug transport, the cornea may be divided into three layers, each of which accounts for the cornea's limited permeability characteristics:

1. The outer epithelium, which is naturally lipophilic.

2. The stroma, which makes up around $90 \%$ of the thickness of the cornea and is hydrophilic,

3. A single layer of flattened epithelium-like cells makes up the inner endothelium.

Because the cornea possesses both hydrophilic and lipophilic components, it acts as a barrier against both hydrophilic and lipophilic chemicals being absorbed.

The nasal cavity, with its bigger surface area and higher permeability of the nasal mucosal membrane than that of the cornea, is another important pathway for the removal of topically administered medicines from the precorneal region.
The nasal mucosal lining, which is continuous with the conjunctival sac, is susceptible to absorption of ocular medicines into systemic circulation 9 .

\section{PRE-OCULAR RETENTION}

The human eye can contain ophthalmic solution without overflow or spilling at the outer angle, however most commercial ophthalmic eye drops dispensers deliver. As a result of the excessive volume administered, a considerable percentage of the medicine is squandered. After the extra solution is removed from the front of the eye, a second process of clearing takes over. The eye has a good tear turnover system. For an instilled solution, the two methods of clearance result in a biphasic profile, with a quick initial clearance phase owing to excess fluid loss followed by a delayed second phase due to tear turnover ${ }^{10}$.

\section{ANATOMY AND PHYSIOLOGY OF EYE}

\section{Anatomy of Eye}

The cornea, conjunctiva, iris, pupil, ciliary body, anterior chamber, aqueous humor, lens, and trabecular meshwork are all part of the anterior segment, whereas the vitreous fluid, sclera, retina, choroid, macula, and optic nerve are all part of the posterior segment. The cornea is the eye's outermost membrane. Epithelium, Bowman's layer, stroma, Descemet's membrane, and endothelium are the five layers that make up this clear, transparent, thin vascular tissue. The clear liquid that fills both the posterior and anterior chambers of the eye is known as aqueous humor. It is the cornea's primary source of nutrition 11 . 


\section{Anatomy of the Eye}

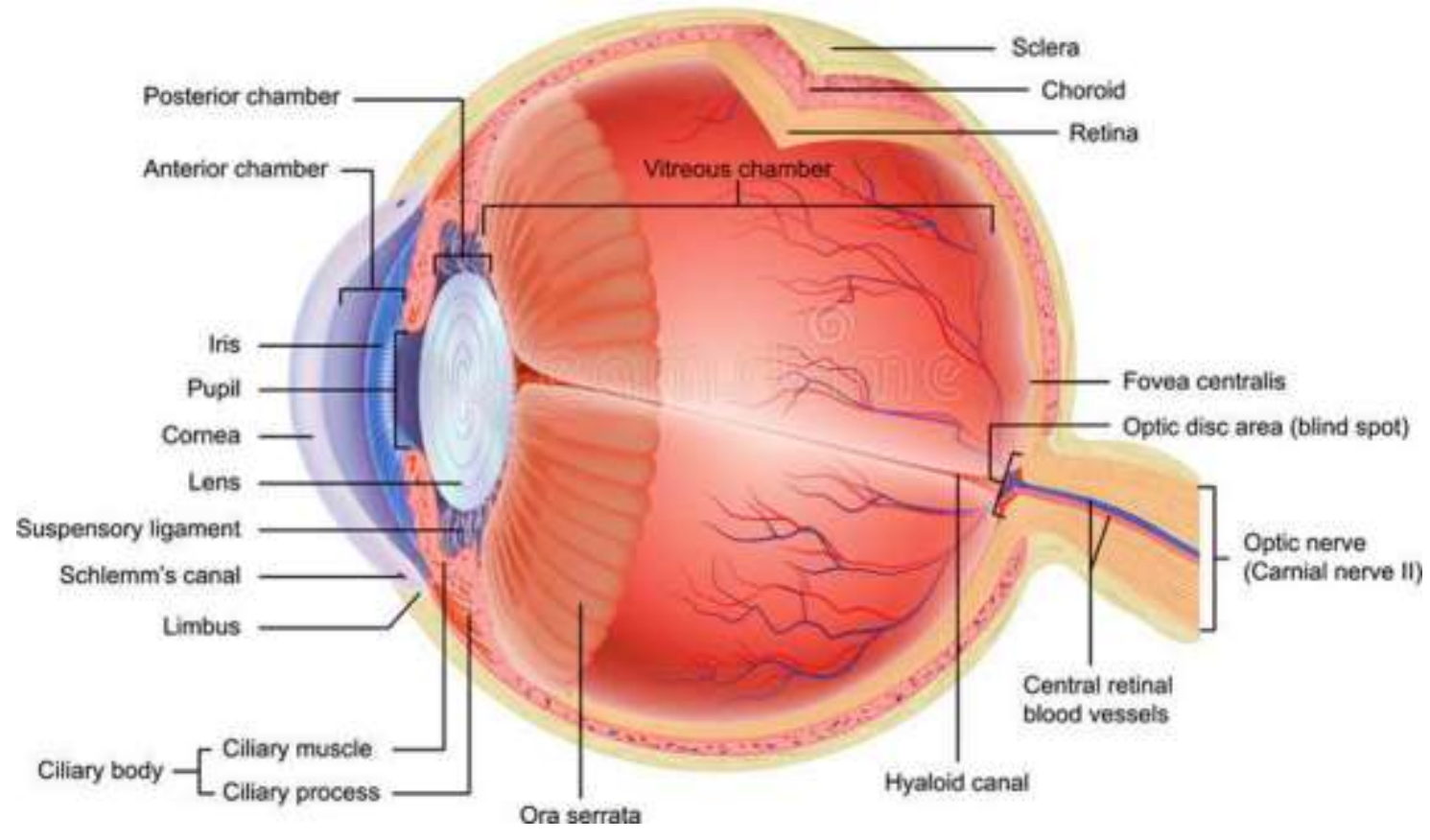

Figure 2: Anatomy of the human eye ${ }^{11}$

- Iris The iris is a thin circular contractile curtain positioned in front of the lens, but below the cornea is a diaphragm of variable size whose role is to regulate the quantity of light admitted into the eye by adjusting the size of the pupil with the aid of the iris sphincter and dilator muscle.

- Ciliary Muscle The ciliary muscle is a ring of smooth muscles in the central layer of the eye that regulates space for viewing objects at different distances.

- Lens The lens is made up of a transparent biconvex structure that is wrapped in a thin transparent coating. It is a flexible entity made up of tissue layers encased in a capsule. The zonules, which are very thin fibers, deflect it from the ciliary muscles ${ }^{12}$.

- Conjunctiva The conjunctiva is a mucous membrane that runs from the border of the cornea to the limbus, lining the interior of the eyelids and sclera. It protects the eyes by secreting mucus that inhibits bacteria from entering and lubricates the eyes.

- Sclera The sclera is the eye's protective outer layer, sometimes known as the "white of the eye," and it keeps the form of the eye. It serves as a first line of defense for the internal organs. The choroid, a highly vascularized tissue wedged between the retina and the sclera, is sandwiched between the sclera and the retina.

- Choroid The choroid is a thin, highly vascular membrane that is dark brown in color and includes a pigment that absorbs excess light, preventing blurry vision. It is the second layer of the eye and is located between the sclera and the retina. It houses the blood arteries that feed the retina's outer layers.

- Retina The retina is made up of vascular, glial, and neuronal cells, as well as nerve fibers, and is a multilayered and complicated structure. It can be found in the human eye's rear chamber. It's a photosensitive structure that captures light rays and converts them to electrical impulses. These impulses pass via the optic nerve and are translated into pictures in the brain.

- Vitreous Chamber The vitreous chamber is a tiny region in front of the eye that includes a watery, thinjelly-like fluid that is spread between the retina and the lens ${ }^{13}$.

\section{ADMINISTRATION OF DRUG TO THE EYE MODE}

As a result, a successful design of a drug delivery system necessitates a comprehensive understanding of the drug molecule. There are a few different ways to get drugs into the ocular tissues ${ }^{14}$. Ophthalmic medication delivery can only be used to treat local eye diseases; it cannot be utilized to introduce a medicine into the systemic circulation. Traditional ophthalmic formulations such as solution, suspension, and ointment have a number of drawbacks that result in low drug bioavailability in the ocular cavity. Ophthalmic medication delivery that is ideal must be able to maintain drug release and stay in the front area of the eye for an extended length of time ${ }^{15}$. Traditional ocular drug delivery methods, such as eye drops, have low bioavailability due to the eye's extensive defensive mechanisms, which make it difficult to reach an effective drug concentration within the target area of the eye. One of the most complicated and a distinctive system in the human body is the anatomy and physiology of the eye. The eye is impervious to foreign substances due to lachrymation, good drainage via the nasolacrimal system, the inner and outer blood-retinal barrier, the cornea's impermeability, and the inability of other non-corneal organs to absorb foreign chemicals 16 . 


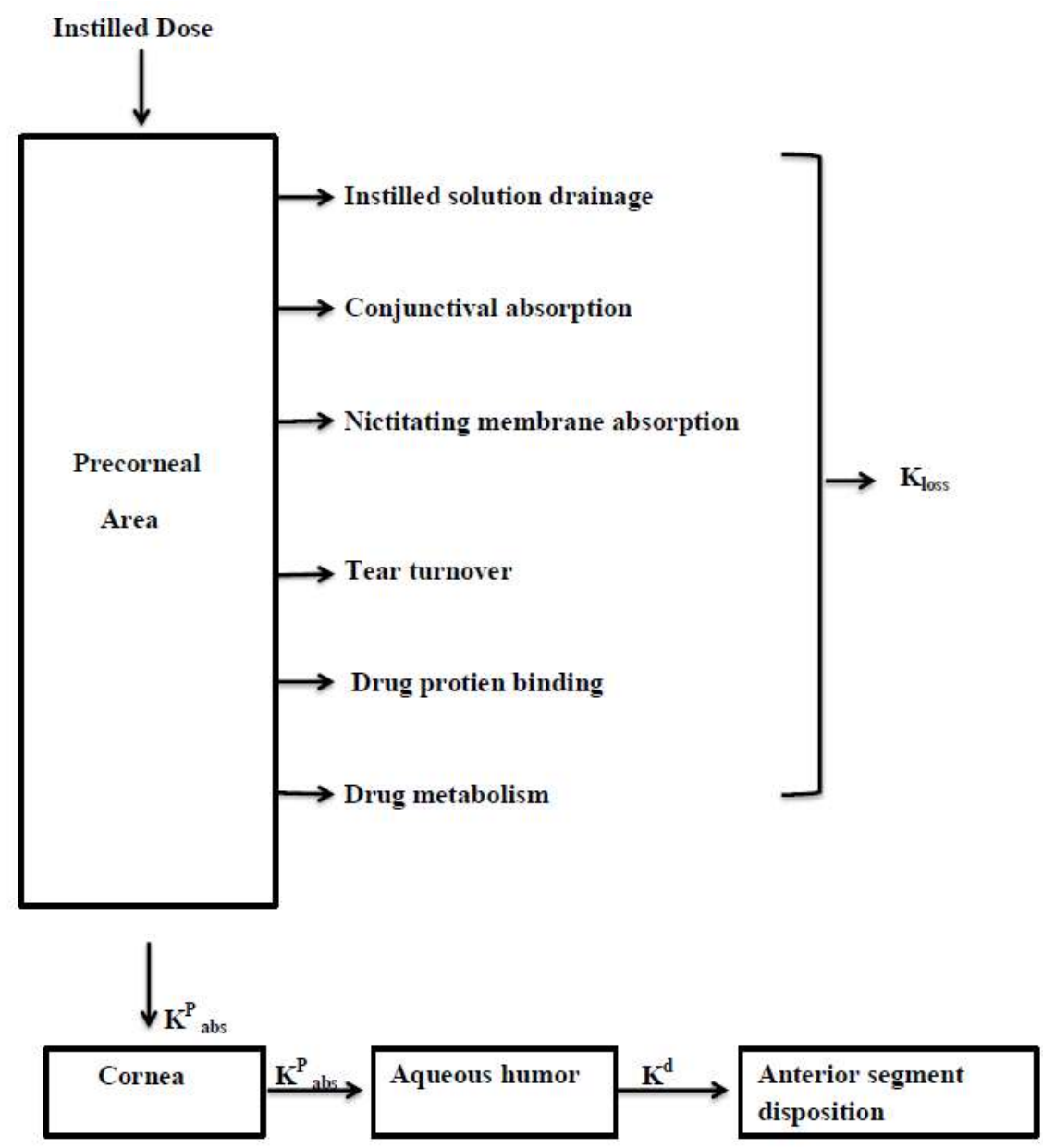

Figure 3: Routes of Administration of drug into Eye 16

\section{CLASSIFICATION OF ADMINISTRATION OF DRUG TO THE EYE MODE-}

1. Topical Administration

2. Oral Administration

3. Systematic Administration

4. Intravitreal Administration

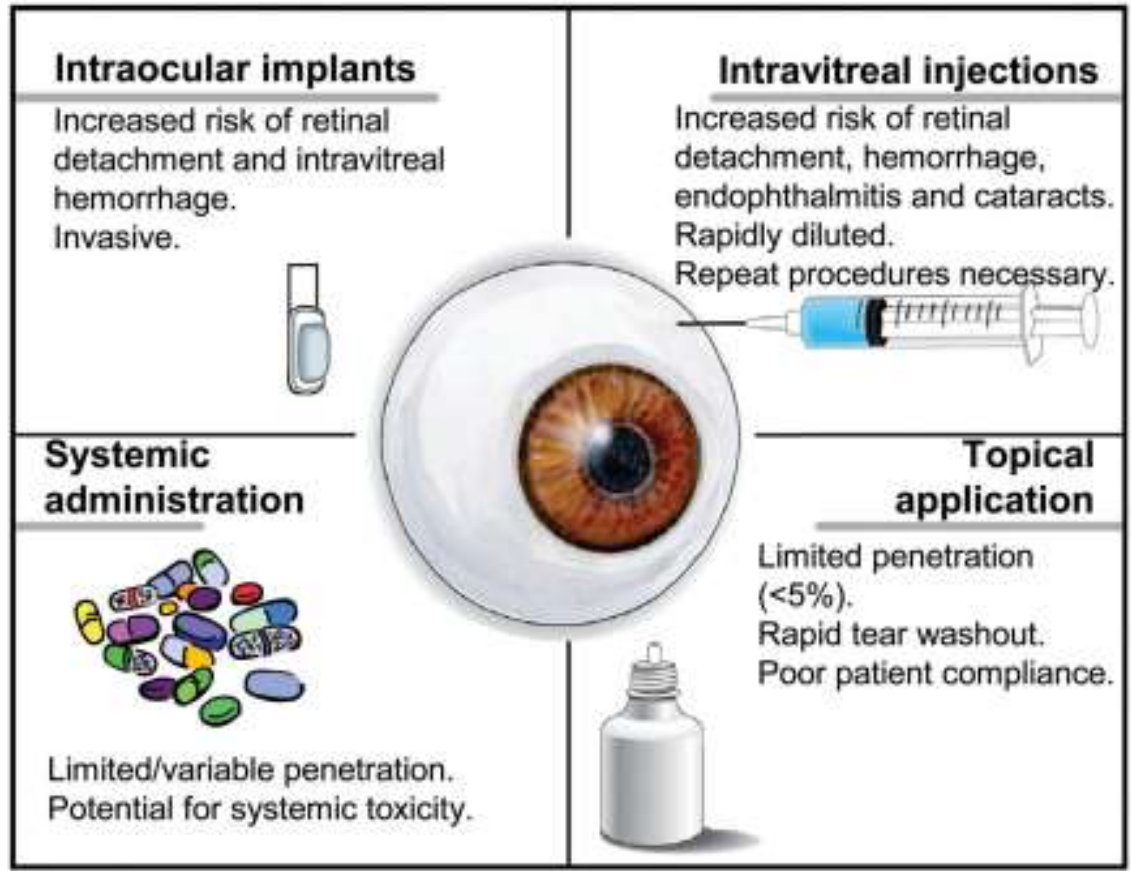

Figure 4: Complications associated with ocular drug delivery ${ }^{17}$. 


\section{Topical Administration}

Eye drops are used for topical ocular medication delivery, although they only have a limited contact period with the eye surface. Gels, jellifying formulations, ointments, and inserts can all help to extend the contact time and hence the duration of pharmacological activity. For most topically administered medications, it is the most regularly utilized method of drug delivery. Different layers of the cornea, conjunctiva, sclera, and other anterior segment tissues such as the iris and ciliary body are typically the sites of action. Precorneal factors and structural barriers reduce the bioavailability of topical formulations after administration ${ }^{18}$.

\section{Oral Administration}

For several reasons, oral administration alone or in conjunction with topical delivery has been researched. In the posterior portion, topical administration failed to generate therapeutic concentrations. In addition, oral administration was compared to parenteral delivery as a patient preferred approach for treating chronic retinal disorders. However, the scarcity of a large number of the targeted ocular tissues limits the utility of oral administration, which necessitates a high dose to achieve significant therapeutic efficacy. Systemic adverse effects may occur as a result of such dosages. As a result, while trying to achieve a therapeutic response in the eye after oral delivery, criteria including safety and toxicity must be assessed 18 .

\section{Systematic Administration}

Following systemic injection, the blood-aqueous and bloodretinal barriers are the principal

Obstacles to ocular drug transport in the anterior and posterior segments, respectively. Systemic administration has had limited effectiveness in delivering medications to the vireo-retinal tissues due to the existence of the blood retinal barrier. This mode of delivery may produce systemic cytotoxicity due to non-specific drug binding to adjacent organs. Even though it is ideal to transport the medicine to the retina by systemic administration, the blood-retina barrier, which tightly limits drug permeability from blood to the retina, remains a hurdle. As a result, unique intravenous targeting mechanisms are required to transport molecules past the choroid and into the retina's deeper layers ${ }^{18}$.

\section{Intravitreal Administration}

Drugs can be administered directly into the vitreous, which allows them to enter the vitreous and retina. Small molecules can flow quickly through the vitreous, while big molecules, especially those that are positively charged, are limited. Because of the RPE (Retinal Pigment Epithelium) barrier, transport from the vitreous to the choroid is more difficult. The distribution of drugs in the vitreous is uneven. This is also dependent on the patho physiological state and the drug's molecular weight. This mode of administration resulted in a longer retention duration and a greater vitreous concentration of medicines ${ }^{14}$.

\section{CRITICAL BARRIERS IN OCULAR THERAPEUTICS}

\section{Drug loss from the ocular surface}

The flow of lacrimal fluid clears the injected substances from the eye's surface after instillation. Despite the fact that the lacrimal turnover rate is only around $1 \mu \mathrm{l} / \mathrm{min}$, the surplus volume of implanted fluid is quickly transported to the nasolacrimal duct [19]. Systemic absorption can occur either immediately from the conjunctival sac through local blood capillaries or after the fluid has passed through the nasal cavity. Regardless, the majority of the administered dosage with a low molecular weight is quickly absorbed into the systemic circulation. The poor ocular bioavailability of less than $5 \%$ is in stark contrast 20.

\section{Lacrimal fluid-eye barriers}

The corneal barrier is generated when the epithelial cells mature. They go from the limbal area to the cornea's center and then to the apical surface [20]. Tight connections occur between the most apical corneal epithelial cells, limiting paracellular drug penetration. As a result, lipophilic medications often have permeability in the cornea that is at least an order of magnitude greater than hydrophilic pharmaceuticals. Trans corneal permeation is the main route of drug entry from the lacrimal fluid to the aqueous humor, despite the tightness of the corneal epithelial layer ${ }^{19}$.

\section{Blood ocular barriers}

Blood-ocular barriers protect the eye from xenobiotics in the bloodstream. The blood-aqueous barrier and the bloodretina barrier are the two types of barriers 19 . The bloodaqueous-barrier (BAB) and the blood-retinal-barrier (BRB) respectively govern the transit of molecules from the systemic circulation to anterior and posterior ocular tissue. Poorly fat-soluble antibiotics' intravitreal drug levels have been found to be less than $10 \%$ of their blood levels ${ }^{21}$.

\section{IN-SITU GEL}

In situ refers to a Latin technique that is literally translated as "in place." In situ gels are drug delivery systems that are introduced into the body prior to being organized, but they go through in situ gelation's to form a gel till they are targeted. For in situ gels, there are five organizational classes: oral, visual, rectal, genital, injectable, and intraperitoneal. The 'in situ gel' framework has been identified as one of numerous new drug transport methods. The in-situ gel framework leads to the supported and regulated arrival of medications, which improves patient quality and comfort with its exceptional 'Sol a Gel' brand of development ${ }^{22}$. A plane that is in a structure of organization before entering the body, but that will convert to a gel form under certain physiological situations. The progression from sol to gel is based on a number of factors, including temperature, $\mathrm{pH}$ variation, solubility trading, UV light, and the closeness of specified atoms or particles. Drug transport facilities with the above-mentioned 'evolution from sol to gel' features are often employed for ongoing planning of vehicles to transport bioactive particles. Some advantages of the "in situ gelling system" include the convenience of dose administration, the decrease of organizational recurrences, and the preservation of pharmaceuticals from changing environmental conditions. In situ gel frames are used to test a range of features and produced polymers, which may be utilized for oral, visual, transdermal, oral, intraperitoneal, parenteral, injectable, rectal, and vaginal applications 23 . Continuous advancements in in-situ gels have made it conceivable to exploit physiological distinctiveness to boost medicine consumption, accommodation, and patient quality in various gastrointestinal tract districts 24, 25. Typical polymers utilized for the in situ gelation structure include gelatin, gylanic acid, chitosan, alginic acid, guar gum, Carbopol, xyloglucan, xanthan gum, HPMC, Poloxamer, and others ${ }^{26}$.

\section{Gel}

Gels are a type of innovative material that combines liquid and solid elements. It is made out of three-dimensional solid networks. Because gels contain a three-dimensional solid network, they are divided into two types based on the structure of the bonds ${ }^{27}$. They are numbered; 
1. The gel network is formed by weak linkages such as hydrogen bonds, electrostatic interactions, and van der Waal bonds.

2. When the gel network has strong covalent connections, chemical gels form. The network demonstrates that cross-links aid in the prevention of hydrophilic polymer breakdown in an aqueous media.

\section{IN-SITU GELLING SYSTEM}

Ophthalmic in-situ gelling is made up of environmentally sensitive polymers that will change structurally in response to tiny changes in environmental factors such as $\mathrm{pH}$, temperature, and ionic strength. In-situ forming gels are liquids that are injected into the eye, then rapidly gel in the cul-de-sac to produce viscoelastic gels in reaction to environmental changes, and then slowly release the medicine under physiological settings ${ }^{28}$. As a result, the insitu gel's residence period is extended, and the medication is delivered in a sustained way, resulting in increased bioavailability, lower systemic absorption, and a less frequent dosage schedule, all of which contribute to greater patient compliance ${ }^{29}$. In-situ gelling devices have also demonstrated several additional potential benefits, such as a simple manufacturing process, convenience of administration, and exact dosage delivery ${ }^{30}$.

\section{IDEAL CHARACTERISTICS OF POLYMERS FOR PREPARATION OF IN SITU GEL 31,32}

1. It is necessary for the mucous membrane to adhere to the polymer.

2. It should be compatible without becoming dangerous.

3. Pseudo plasticity is required.

4. The cutting speed of the polymer can be increased to reduce viscosity.

5. In situ gel preparation requires a high level of tolerance and optical clarity.

6. Tear behavior must be influenced.

\section{ADVANTAGES OF IN SITU GEL SYSTEM 33, 34}

1. Drug release that is both controlled and prolonged.

2. Administering drugs quickly.

3. Can be administered to patients who are unconscious.

4. It increased patient comfort and compliance.

5. Lower the dose and the toxicity of pharmaceuticals.

6. Bioavailability has been improved.

7. Biocompatible and biodegradable natural polymers

8. Natural polymers enhance cellular function due to their intrinsic biocompatibility, biodegradability, and physiologically recognizable components.

9. Synthetic polymers have well-defined structures that can be tweaked to provide acceptable functionality and degradability.

10. In situ gels can also be used to show adherence while collecting medical materials for non-invasive medication administration, such as via mucosal membranes.

11. Because of their hydrophilicity, in situ gels play a vital role in vivo, extending the circulation duration of the invivo release device by avoiding the host's immunological response and reducing phagocytic activity.

\section{DISADVANTAGES OF IN SITU GEL SYSTEM 35, 36}

1. Requires a lot of fluid.

2. The drug's solar form is more biodegradable.

3. Degradation of the chemical may cause stability difficulties.

4. For a few hours after taking the medicine, eating and drinking are limited.

5. The volume and homogeneity of the drug load in hydro gels can be lowered, especially in hydrophobic pharmaceuticals.

6. Only low-dose medications are permitted. It needs a large number of fluids.

Polymers used in temperature triggered in-situ gel systems $37,38,39,40,41$

- Poloxamers (Pluronic)

- Xyloglucan

- Cellulose derivatives

- Chitosan

- Carbopol

EVALUATION PARAMETERS OF IN SITU GEL SYSTEM 42,43

There is various type of evaluation parameters in situ gel system such as:

1. $\mathrm{pH}$

2. Clarity

3. Texture Analysis

4. Gelling Capacity

5. Gel Strength

6. Rheological Studies

7. Sol-Gel Transition Temperature

8. Fourier Infrared Spectroscopic Transforms

1. pH

The $\mathrm{pH}$ of the gel was measured with a calibrated $\mathrm{pH}$ meter, and values were collected for three samples on average.

\section{Clarity}

Examining each container in excellent light, looking for reflections into the eyes, and displaying against a dark and bright background are all part of the clarity test.

\section{Texture Analysis}

The formulation's purity and durability were assessed using a texture analyzer, which largely displays the sol's syringe ability, allowing the formulation to be delivered in vivo with ease. To maintain tight contact with a tissue-like surface, higher adhesive values for gels are required.

\section{Gelling Capacity}

The gelling capacity of the formula is evaluated by placing drops in a vial containing $2 \mathrm{~mL}$ of newly generated simulated tear fluid and visually inspecting it. It is observed how long it takes for the gel to form.

\section{Gel Strength}


Rheometer is used to measure this parameter. Depending on the mechanism of the gelling agent, the beaker prepares a certain quantity of gel from the sol type. The collateralized on the sample may be determined as a function of the sample's immersion depth below the gel surface on this beaker gel, which is set at a level that allows a sample to flow slowly through the gel.

\section{Rheological Studies}

Viscosity tests are carried out with a Brookfield programmed DVII+Model pro II type viscometer (USA). In the sampler tube, the in-situ gel formulations are placed. Before each measurement, circulated baths linked to the viscometer adapter are evaluated at $37{ }^{\circ} \mathrm{C} 0.5{ }^{\circ} \mathrm{C}$. The formulation viscosity is determined after increasing the spindle angular velocity from 1 to 4 .

\section{Sol-Gel Transition Temperature}

The temperature at which the fluid transition phase becomes a gel may be used to establish the sol-gel transition temperature. When test tubes are tilted at a 90-degree angle with a continuous temperature rise, the gelation point is the temperature at which compositions will not flow. The $\mathrm{pH}$ and ion-dependent polymers change from sol to gel when a $\mathrm{pH}$ or nasal fluid transition is present.

\section{Fourier Infrared Spectroscopic Transforms}

An FT-IR spectrometer is used to create the Fourier infrared transform (FT-IR) spectrometer. The active drug was forcefully mixed with a predetermined ratio of potassium bromide and a clear infrared matrix. (Typically, the ratio is $1: 5)$

\section{ACKNOWLEDGEMENT}

I am very thankful to principal, Shivalik College of pharmacy, Nangal, Punjab and my guide Dhruv sir for their valuable guidance. I am also thankful to my colleagues for their timeto-time support.

\section{CONCLUSION}

As previously said in this analysis, the eye is one of the most complex and sophisticated organs. Many improvements have been obtained in anterior DDSs (drug delivery system) for extending retention period and lowering administration frequency, however further requirements are needed in this area. It's possible that the field may improve in terms of patient care and compliance. On the other hand, there are a few new items on the market. As a result of the study, a number of ophthalmic delivery systems have been marketed. These new goods' performance, on the other hand, is still far from satisfactory. An ideal ocular drug delivery system would be able to maintain the lowest effective drug concentration in the target tissue of the eye for a long time while limiting systemic exposure and it would also be pleasant to use. In each of the technologies included in this study, more research is necessary. Some formulations for ocular delivery systems are relatively simple to make, but they have limitations in terms of their capacity to offer sustained and regulated drug release over lengthy periods of time. Other techniques, including as particulates, liposomes, oligonucleotide therapy, aptamer, and other emerging advanced delivery systems, are promise in terms of prolonged and regulated drug release, but they are challenging to make, utilize, and achieve Stability. The revolutionary improved delivery technologies provide a more protective and effective way of therapy for illnesses of the eyes that are practically unreachable. The most recent targeted drug delivery systems concentrate on delivering medications and certain macromolecular substances such as DNA, siRNA, and protein to the eye's interior components in a safe and convenient manner.

\section{REFERENCES}

1. EL-Kamel AH. In vitro and in vivo evaluation of Pluronic F127based ocular delivery system for timolol maleate. International journal of pharmaceutics 2002; 24 (1):47-55. https://doi.org/10.1016/S0378-5173(02)00234-X

2. Varshosaz J, Tabbakhian M, Salmani Z. Designing of a Thermo sensitive Chitosan/Poloxamer In Situ Gel for Ocular Delivery of Ciprofloxacin. The Open Drug Delivery Journal 2008; 2:61-70. https://doi.org/10.2174/1874126600802010061

3. Nalla A and Chinnala K M. In - situ ophthalmic drug delivery systems - An Overview, Indo Am. J. Pharm. Sci. 2016; 3(3): 202208.

4. Peppas NA, Langer R. New challenges in biomaterials. Science 1994; 263(5154):1715-1720. https://doi.org/10.1126/science.8134835

5. Swapnil S. A review on polymers used in novel in situ gel formulation for ocular drug delivery and their evaluation. Journal of biological and scientific opinion 2003; 1(2):132-137. https://doi.org/10.7897/2321-6328.01221

6. Mishima, S., Gasset, A., Klyce, S.D., Baum, J.L. Determination of tear volume and tear flow. Invest. Ophthalmol1966; 5:264-276.

7. Watzke M.A., Jablonski M.M., Edelhauser H.F. Comparison of conjunctiva and corneal surface areas in rabbits and human. Curr. Eye Res.1988; 7:483-486. https://doi.org/10.3109/02713688809031801

8. Lee V.H.-L., Robinson J.R. Mechanistic and quantitative evaluation of precorneal pilocarpine disposition in albino rabbits. J. Pharm. Sci.1979; 68:673-684. https://doi.org/10.1002/jps.2600680606

9. Desai S.D., Blanchard J., Swarbick J., Boylan J.C. (Ed.), Ocular drug formulation and delivery. Encyclopedia of Pharmaceutical Technology, Marcel Dekker, New York, 1944; 11:43-75.

10. Chrai S.S., Patton T.F., Mehta A., Robinson J.R., 1973. Lacrimal and instilled fluid dynamics in rabbit eyes. J. Pharm. Sci.1973; 62:1112-1121. https://doi.org/10.1002/jps.2600620712

11. Yumei WU et al., Research Progress of in-situ gelling Ophthalmic Drug Delivery System, Asian Journal of Pharmaceutical Science, 2018; 22-40.

12. Hajare A, Mali S, Salunke S, Nadaf S, Bhatia N, Bagal P, Gaikwad S, Pawar K, A Rational Approach to Ocular Drug Delivery System: An Overview, World Journal of Pharmaceutical Science, 2014; 3(2):3324-3348.

13. Yerikala R, Kothapalli CB, Peddappi Reddigari JR, A Novel Approach on Ophthalmic Drug Delivery System, Journal of Drug Delivery and Therapeutics, 2017; 7(6):117-124. https://doi.org/10.22270/jddt.v7i6.1512

14. Pandya TP, Modasiya MK, Patel VM, Ophthalmic In-Situ Gelling System, International Journal of Pharmacy and Life Sciences, 2011; 2(5): 730-738.

15. Jain S, Jain P, Mishra M, Pathak A, A Review on Triggered Gel for Ocular Drug Delivery System, International Journal of Pharmaceutical and Biological Archieves, 2014; 5(4):19-24.

16. Palani S, Joseph NM, Goda CC, Zachariah A, Ayenew Z, Ocular Drug Delivery: A Review, International Journal Pharmaceutical Science and Research, 2010; 1(3):1-11.

17. Short BG. Safety evaluation of ocular drug delivery formulation: techniques and practical considerations. Toxicologic pathology, 2008; 36:49-62. https://doi.org/10.1177/0192623307310955

18. Saini N, Kumar D, An Insight to Ophthalmic Drug Delivery System, International Journal of Pharmaceutical Studies and Research 2012; 3(2):09-13. 
19. Pandey H, Sharma UK and Pandey AC. Eudragit- based nanostructures: A potential approach for ocular drug delivery. Int J Res Dev Pharm L Sci, 2012; 1(2):40-3.

20. Tangri $P$, and Khurana S. basics of ocular drug delivery systems. Int J Res Pharm and Biomed Sci 2011; 2(4):1541-1552.

21. Haders DJ. New controlled release technologies broaden opportunities for ophthalmic therapies. Drug delivery technology, 2008; 8(7):48-53.

22. Patel Nisha, Shinde Gajanan, and KS Rajesh. Ophthalmic In situ gel, A genesis journal Pharmagene 2014; 2(4):29-33.

23. Suisha F, Kawasaki N, Miyazaki S, Shirakawa M, Yamatoya K, Sasaki M, Attwood D, Xyloglucan gels as sustained-release vehicles for the intraperitoneal administration of mitomycin C. Int. J. Pharm., 1998; 172:27- 32.

https://doi.org/10.1016/S0378-5173(98)00157-4

24. Miyazaki S, Endo K, Kawasaki N, Kubo W, Watanabe H, Attwood D. Oral sustained delivery of paracetamol from in situ gelling xyloglucan formulations. Drug Dev Ind. Pharm. 2003; 29(2):1139. https://doi.org/10.1081/DDC-120016718

25. Nerkar Tushar, Gujarathi Nayan A, Rane Bhushan R, Bakliwal Sunil R, Pawar S.P. In situ gel: Novel Approach in a sustained and controlled drug delivery system. International Journal of Pharmaceutical Sciences 2013; 4(4):1-18.

26. Saraswat R.1, Bhan C. S., Gaur A. A Review on Polymers Used in In-Situ Gel Drug Delivery Systems 2011; 1(2).

27. Zhidong L, Jaiwei L, Shufang N, Study of a Pharma alginate- HPMC based in situ gelling ophthalmic delivery system for gatifloxacin. Int J., 2016; 315:12- 7.

28. Khan N, AqilM, ImamSS, Ali A. Development and evaluation of a novel in situ gel of sparfloxacin for sustained ocular drug delivery: in vitro and ex vivo characterization. Pharm Dev Technol 2015; 20(6):662-9 https://doi.org/10.3109/10837450.2014.910807

29. Li J, ZhaoH, OkekeCI, etal. Comparison of systemic absorption between ofloxacin ophthalmic in situ gels and ofloxacin conventional ophthalmic solutions administration to rabbit eyes by HPLC-MS/MS. Int J Pharm 2013; 450(1-2):104-13. https://doi.org/10.1016/j.ijpharm.2013.04.018

30. Devasani SR, DevA, RathodS, DeshmukhG. An overview of in situ gelling systems. Pharmaceut Biolog Evaluat 2016; 3(1):60-9.

31. Srividya B, Cardoza R. M., Amin P.D. Sustained ophthalmic delivery of ofloxacin from a $\mathrm{pH}$ triggered in situ gelling systems.
J. Control Release. 2001; 73:205-211.

https://doi.org/10.1016/S0168-3659(01)00279-6

32. Wen-Di Ma, Hui Xu, Chao Wang, Shu-Fang Nie, Wei-San Pan, Pluronic F127-g-poly (acrylic acid) copolymers as in situ gelling vehicles for ophthalmic drug delivery system, int. j. of pharmaceutics, 2008; (350):247-256. https://doi.org/10.1016/j.ijpharm.2007.09.005

33. Vodithala Sirish, Khatry Sadhana, Shastri Nalini, Sadanandam M, Formulation and evaluation of ion activated ocular gels of ketorolac tromethamine International Journal of Current Pharmaceutical Research, $2010 ; 2(3)$.

34. M. Jothi, Harikumar SL and Aggarwal Geeta, In-situ ophthalmic gels for the treatment of eye diseases, International Journal of Pharmaceutical Sciences and Research, 2012; 3:1891-1904.

35. Rajas NJ, Kavitha K, Gounder T, Mani T, In-Situ ophthalmic gels a developing trend, Int J Pharm Sci Rev and Res, 2011; 7:8-14.

36. Geraghaty P, Attwood D, et al. An investigation of parameters influencing the Bioadhesive properties of Myverol 18-99/ water gels. Biomaterials 1997; 18:63-7. https://doi.org/10.1016/S0142-9612(96)00087-7

37. Devasani SR, DevA, RathodS, Deshmukh G. An overview of in situ gelling systems. Pharmaceut Biolog Evaluat 2016; 3(1):60-9.

38. Laddha UD, Mahajan HS. An insight to ocular in situ gelling systems. Int J Adv Pharmaceut 2017; 06(02):31-40.

39. Klouda L. Thermoresponsive hydrogels in biomedical applications: a seven-year update. Eur J Pharm Biopharm 2015;97(Pt B):338-49. https://doi.org/10.1016/j.ejpb.2015.05.017

40. Zambito Y, Colo GD. Polysaccharides as excipients for ocular topical formulations. Polysaccharides as excipients for ocular topical formulations. InTech; 2011. p. 253-80. https://doi.org/10.5772/24430

41. Mohammed S, Chouhan G, Anuforom O, et al. Thermosensitive hydrogel as an in situ gelling antimicrobial ocular dressing. Mater Sci Eng C Mater Biol Appl 2017; 78:203-9. https://doi.org/10.1016/j.msec.2017.04.065

42. Sandeep DS, Charyulu NR, Narayanan AV, Smart in Situ Gels for Glaucoma-An Overview, International Journal of Pharmaceutical Science Research and Review, 2018; 50(1):94-100.

43. Majeed A., Khan N. A. Ocular in situ gel: An overview. Journal of Drug Delivery and Therapeutics, 2019; 9(1):337-347. https://doi.org/10.22270/jddt.v9i1.2231 